\title{
Hemangioendotelioma kaposiforme e síndrome de Kasabach-Merritt
}

\author{
Kaposiform haemangioendothelioma and Kasabach-Merritt syndrome
}

\author{
Adriana de Oliveira Mukai', Ana Glenda S. Zanlochi², Chelna Paolichi F. Elias², Christine Holtz Rolim², Luciana Lucatto Baida², Patrícia \\ Cavalcante Tutia ${ }^{2}$, Ricardo Marcitelli ${ }^{1}$
}

\section{RESUMO}

Objetivo: Descrever a apresentação e a evolução de um caso de hemangioendotelioma kaposiforme complicado por síndrome de Kasabach-Merritt.

Descrição do caso: Lactente masculino de 45 dias, com febre, irritabilidade e choro, associados ao aparecimento de nodulações em tronco e membros. Evoluiu rapidamente com coagulopatia de consumo e queda do estado geral, necessitando de internação em Unidade de Terapia Intensiva Pediátrica (UTIP). Tomografia computadorizada revelou condensações parenquimatosas intra e extrapulmonares compatíveis com hemangiomas múltiplos e ausência de lesões em crânio e abdome. Houve complicação para síndrome Kasabach-Merritt em conseqüência de uma hemangiomatose kaposiforme. Optado por iniciar tratamento conservador, associando terapia de suporte com concentrado de glóbulos vermelhos, criopreciptado, plasma fresco, concentrado de plaquetas e uso das seguintes drogas: ácido tranexâmico e dexametasona. Após 20 dias em UTIP, já se encontrava em bom estado geral, em processo de remissão dos hemangiomas e normalização dos exames laboratoriais, sendo transferido para a enfermaria. Recebeu alta em tratamento com ácido tranexâmico e prednisona, com acompanhamento ambulatorial e laboratorial semanal.

Comentários: A doença apresenta curso clínico rápido e grave, com necessidade de diagnóstico e tratamento precoces, para melhor prognóstico e sobrevida.

Palavras-chave: hemangioendotelioma; síndrome de Kasabach-Merritt; coagulação intravascular disseminada.

\section{ABSTRACT}

Objective: To describe the clinical presentation of kaposiform hemangioendothelioma that was complicated by Kasabach-Merritt syndrome.

Case description: A 45-day-old male infant presented fever, irritability and crying associated with the eruption of nodules in trunk and members. Soon after admission, the patient developed a consumptive coagulopathy, requiring admission in the Pediatric Intensive Care Unit (PICU). Computed tomography scans showed intra and extrapulmonary condensations suggestive of multiple thoracic hemangiomas, without the central nervous system and abdominal lesions. The diagnosis of KasabachMerritt syndrome in consequence of kaposiform hemagioendothelioma was established. Treatment option was conservative, including red blood cells, cryoprecipited, fresh frozen plasma and platelets transfusions associated to tranexamic acid and dexamethasone. After 20 days, patient recovered and he was discharged from PICU with significant remission of the hemangiomas. The child continued to receive tranexamic acid and prednisone at home, with weekly follow-up.

Comments: The disease presents quick and severe clinical course. Therefore, early diagnosis and treatment are needed to improve the outcome.

Key-words: haemangioendothelioma; Kasabach-Merritt syndrome; disseminated intravascular coagulation.
${ }^{1}$ Mestres em Ciências pela Faculdade de Medicina da Universidade de São Paulo (FMUSP) e professores-assistentes de Pediatria do Departamento de Medicina da Universidade de Taubaté (Unitau), Taubaté, SP, Brasil

${ }^{2}$ Acadêmica do Departamento de Medicina da Unitau, Taubaté, SP, Brasil
Endereço para correspondência:

Chelna Paolichi F. Elias

Rua Padre Diogo Antônio Feijó, 125, apto. 12 - Centro

CEP 12030-160 - Taubaté/SP

E-mail: med_chelna@yahoo.com.br 


\section{Introdução}

Hemangiomas são tumores vasculares congênitos que apresentam, na população geral, prevalência de 2 a $6 \%$, sendo os tumores mais comuns na infância ${ }^{(1)}$. Possuem rápido crescimento pós-natal, o qual pode perdurar por vários meses (fase proliferativa) e se estender até os 18 meses de idade, quando a maioria começa a sofrer involução. Os hemangiomas desaparecem entre cinco e oito anos de idade em $70 \%$ dos casos, sem deixar seqüelas. Estes tumores vasculares podem ser superficiais ou apresentar envolvimento de vísceras ${ }^{(2)}$.

O hemangioendotelioma kaposiforme é um tumor raro, de origem vascular, que predomina na infância. Tem características histológicas benignas, porém apresenta comportamento maligno com proliferação local e, freqüentemente, agressão vascular. A afecção envolve pele, subcutâneo e tecidos moles mais profundos, atingindo até retroperitônio, em alguns $\operatorname{casos}^{(3)}$. Metástases à distância não são relatadas. A associação com proliferação do sistema linfático é comum e tem forte associação com a síndrome de Kasabach-Merritt ${ }^{(4)}$.

A síndrome de Kasabach-Merritt consiste de hemangioma proliferativo extenso, que leva à coagulopatia de consumo. Em geral, não há outras malformações congênitas associadas. A síndrome foi descrita em 1940 como uma associação entre "hemangiomas capilares" e trombocitopenia ${ }^{(5)}$. Na realidade, ocorrem hemangiomas tuberosos extensos, que evoluem rapidamente no primeiro ano de vida e se acompanham de equimoses e petéquias no corpo, determinadas pela coagulopatia de consumo. A trombocitopenia está associada ao aprisionamento das plaquetas no interior da malformação vascular. A instalação do quadro pode ocorrer durante o processo de crescimento do hemangioma ou, mais tardiamente, associada à contusão da lesão. O diagnóstico é clínico e laboratorial, com número de plaquetas em torno de $10.000 / \mathrm{mm}^{(3,6)}$.

O objetivo deste trabalho foi apresentar o relato de caso de um lactente masculino de 45 dias com hemangioendotelioma kaposiforme associado à síndrome de Kasabach-Merritt, por se tratar de complicação grave, em que o reconhecimento precoce de sua apresentação clínica e laboratorial auxilia o tratamento e o prognóstico, além de possibilitar melhor sobrevida.

\section{Descrição do caso}

Lactente de 45 dias, masculino, branco, natural e procedente de Taubaté, São Paulo. Na semana anterior à admissão, apresentou pico febril, evoluindo com irritabilidade e choro freqüente. Negava vômitos ou diarréia no período. Quatro dias antes do início do quadro, houve dificuldade na movimentação do membro superior direito, com choro intenso à manipulação do mesmo, associado à diferença na coloração e diminuição da temperatura do membro em relação ao restante do corpo, além da presença de edema. Negava traumas anteriores. Um dia antes, houve aparecimento de um abaulamento na altura do apêndice xifóide, em local onde previamente existia uma mancha congênita, além do surgimento de outras pequenas nodulações em dorso da mão, punho e axila direitos.

Ao exame clínico, encontrava-se em regular estado geral, as mucosas eram úmidas e descoradas, estava afebril, gemente, hipoativo, reativo. O peso era de $5.260 \mathrm{~g}$ (percentil 90 do peso para a idade) e estatura de $54 \mathrm{~cm}$ (percentil 50 do comprimento para a idade). A inspeção da pele mostrava palidez cutânea, pápula eritematosa em punho esquerdo, nódulo vinhoso de aproximadamente $2 \mathrm{~cm}$ de diâmetro em região esternal, associado a sinais flogísticos: hiperemia e edema em ombro, tornozelo e joelho direitos, e pequena pápula em hálux direito (Figura 1). Havia dificuldade na abdução e rotação do membro superior direito e o reflexo de Moro era ausente à direita.

No primeiro dia de internação, apresentou evento súbito de cianose central e apnéia, evoluindo com parada cardiorrespiratória. O paciente recebeu manobras de reanimação, sendo intubado por obstrução de vias aéreas com cânula orotraqueal e administrada adrenalina, com resposta satisfatória após sete minutos. A criança foi transferida para a Unidade de Terapia Intensiva Pediátrica (UTIP), colocada em ventilação mecânica e iniciadas drogas vasoativas (dopamina e dobutamina) devido à instabilidade hemodinâmi$\mathrm{ca}$, sendo estas medicações mantidas por sete dias. $\mathrm{O}$ paciente evoluiu com anemia (hemoglobina de 7,6g/dL e hematócrito de $22,3 \%)$, plaquetopenia $\left(23.000 / \mathrm{mm}^{3}\right)$, prolongamento do

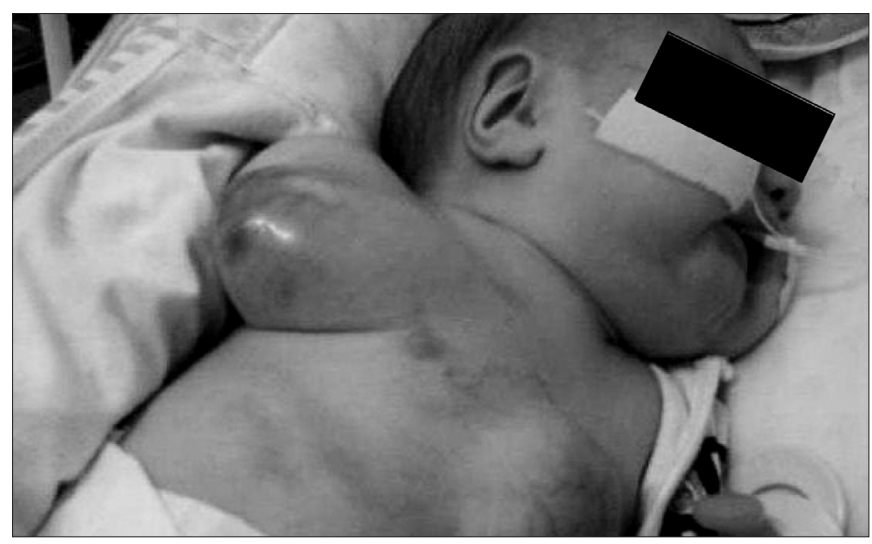

Figura 1 - Apresentação do paciente à admissão hospitalar. Notar mancha esternal e hiperemia e edema em ombros e cotovelo direito. 
tempo de tromboplastina parcial ativada (TTPA), da atividade de protrombina (AP) e com consumo de fibrinogênio. Introduzido oxacilina e ceftriaxone.

Realizados exames de imagem pela possibilidade de lesões semelhantes em órgãos viscerais. Na tomografia computadorizada (TC) de crânio e de abdome não foram encontradas alterações. Na TC de tórax, havia presença de condensações parenquimatosas nos segmentos pulmonares posteriores e opacidade de contornos lobulados, medindo $1,2 \times 1,0 \mathrm{~cm} \mathrm{e}$ $2,5 \times 1,0 \mathrm{~cm}$, em ambas as bases pulmonares, em terço superior e anterior do hemitórax direito e na base do esquerdo. Foram também visualizadas outras lesões semelhantes extratorácicas, na linha média, medindo $2,4 \times 1,5 \mathrm{~cm}$. Notaram-se hilos pulmonares anatômicos e superfícies pleurais lisas, com aumento do volume de ombro direito à custa de imagem hipodensa (líquido espesso), na topografia da artéria gleno-umeral.

No segundo dia de evolução, houve piora do estado geral, com anasarca e aumento das lesões torácicas e de axila direita. Após avaliação da Hematologia do quadro de hemangiomas múltiplos e plaquetopenia, foi feito o diagnóstico de síndrome Kasabach-Merritt em conseqüência de hemangiomatose kaposiforme, sendo introduzida terapia com ácido tranexâmico $(200 \mathrm{mg} / \mathrm{kg} / \mathrm{dia})$ e dexametasona $(1 \mathrm{mg} / \mathrm{kg} / \mathrm{dia})$.

Houve aparecimento de lesões petequiais em tronco e outros hemangiomas em joelho esquerdo e punho direito, no terceiro dia de internação. Realizados novos exames laboratoriais: hemoglobina de $10,7 \mathrm{~g} / \mathrm{dL}$, hematócrito $29,7 \%$, plaquetas de $42.000 / \mathrm{mm}^{3}$, AP de $50 \%$, TTPA de 60 segundos e fibrinogênio de $1,5 \mathrm{mg} / \mathrm{dL}$, compatíveis com coagulação intravascular disseminada (CIVD). Realizou-se transfusão de plasma fresco e de crioprecipitado. Os exames pós-transfusionais foram: hemoglobina de $9,5 \mathrm{~g} / \mathrm{dL}$, hematócrito de $28,5 \%$, plaquetas de $28.000 / \mathrm{mm}^{3}$, AP de $92 \%$, TTPA de 49 segundos e fibrinogênio de $2,13 \mathrm{mg} / \mathrm{dL}$.

A partir do quarto dia de evolução, manteve-se o uso do ácido tranexâmico e dexametasona associado à realização de transfusões seqüenciais de hemoconcentrado por cinco vezes, criopreciptado por sete vezes, plasma fresco por três vezes e concentrado de plaquetas por quatro vezes, até o $11^{\circ}$ dia de evolução. Notou-se resposta satisfatória, com estabilidade hemodinâmica e respiração espontânea.

Após 12 dias de internação, foram realizadas novas TC de abdome e tórax devido à possibilidade de desenvolvimento de hemangiomas viscerais. A TC de abdome não revelou alterações. Na TC de tórax, houve aumento significativo das lesões em relação à anterior; todas com contornos regulares, bem delimitados e de densidade líquida, sendo a axilar direita de $5,3 \times 2,9 \mathrm{~cm}$, a parietal anterior de $4,2 \times 4 \mathrm{~cm}$ com componente intratorácico comprimindo face anterior cardíaca e a intratorácica ântero-lateral direita com $8 \times 1,5 \mathrm{~cm}$. Permaneciam as condensações parenquimatosas pulmonares nos segmentos pulmonares posteriores.

No $15^{\circ}$ dia de evolução, apresentou piora clínica, com evidência de sepse (taquicardia, taquipnéia e febre), cujo foco era um abscesso sobreposto ao hemangioma axilar direito. Os exames séricos demonstraram leucócitos de 32.000/ $\mathrm{mm}^{3}$, plaquetas de $367.000 / \mathrm{mm}^{3}$, AP de $116 \%$, TTPA de 29 segundos e fibrinogênio de $1,25 \mathrm{mg} / \mathrm{dL}$. O abscesso foi drenado, havendo melhora clínica importante. A cultura da secreção mostrou crescimento de Enterobacter sp. Suspensa a oxacilina e introduzidos vancomicina e imipenem. Nesse período, foi suspensa a dexametasona e substituída por prednisona $(2 \mathrm{mg} / \mathrm{kg} / \mathrm{dia})$.

Após 20 dias em UTIP, o paciente já se encontrava em bom estado geral, em processo de remissão dos hemangiomas e normalização das provas de coagulação, sendo transferido para enfermaria. Recebeu alta depois de 15 dias, em tratamento com ácido tranexâmico $(200 \mathrm{mg} / \mathrm{kg} / \mathrm{dia})$ e prednisona $(2 \mathrm{mg} / \mathrm{kg} / \mathrm{dia})$, com orientação para acompanhamento ambulatorial e laboratorial semanal.

No retorno ao Ambulatório de Hematologia Infantil após dois meses, observou-se remissão quase total dos hemangiomas e o coagulograma manteve-se normal. Com a melhora significativa do quadro, iniciou-se a redução da corticoterapia e do antifibrinolítico, programados até a suspensão total da medicação. Devido à mudança de endereço da família, o paciente não mais retornou ao ambulatório.

\section{Discussão}

O hemangioendotelioma kaposiforme é um tumor raro e agressivo, sendo que menos de 100 casos foram relatados na literatura desde a sua descrição, em $1993^{(7)}$. O tumor possui características histológicas benignas, porém leva a alta mortalidade na ausência de tratamento. Acomete, em geral, os menores de dois anos e tem incidência levemente aumentada em meninas ${ }^{(1)}$. Desenvolve-se como lesão única na pele, sendo mais comum em tronco ou extremidades. Os tumores crescem até 18 meses de vida $^{(2)}$, semelhante ao relato de caso apresentado.

Nos Estados Unidos, os hemangiomas ocorrem em cerca de 2,5\% dos neonatos. A maioria é benigna e 70 a $80 \%$ regridem entre cinco e oito anos de idade. Um a cada 300 hemangiomas apresenta coagulopatia como complicação ${ }^{(2)}$, sendo freqüente a associação com linfangiomatose ou síndrome de 
Kasabach-Merritt ${ }^{(8)}$. Porém, na atualidade, a maioria dos casos de hemangioendoteliomas kaposiformes é diagnosticada antes do aparecimento de suas complicações ${ }^{(9)}$. No Brasil, não está bem estabelecida a frequiência desta doença.

A síndrome de Kasabach-Merritt é uma coagulopatia de consumo associada à presença de grande lesão vascular, mais comum em lactentes jovens. Sua fisiopatologia não está bem determinada. Estima-se que o rápido consumo de plaquetas e fibrinogênio nos capilares do hemangioma constitui a raiz da CIVD, causa principal de óbito dos pacientes acometidos ${ }^{(8)}$. A apresentação clínica caracteriza-se por presença de hemangiomas cutâneos isolados ou múltiplos e, em sua maioria, localizados em extremidades. Alguns pacientes possuem formas viscerais (fígado, baço, pulmão, cérebro e intestino). Assim, a presença das lesões em tais órgãos sempre deve ser pesquisada por meio de TC ou ressonância magnética de crânio, tórax e abdome $^{(6)}$. Quando os hemangiomas cutâneos não estão presentes, deve-se procurar por hemangiomas viscerais diante de quadro de coagulopatia ${ }^{(4)}$.

O comprometimento cardiovascular pode ocorrer e evoluir com insuficiência cardíaca congestiva e/ou colapso cardiovascular $^{(2)}$. No quadro inicial, a trombocitopenia e a coagulopatia de consumo não são proeminentes. Entretanto, conforme o hemangioma cresce, os lactentes tendem a piorar. O aparecimento de petéquias e sangramento franco é o sintoma inicial que, normalmente, leva à suspeita diagnóstica e à abordagem terapêutica ${ }^{(6)}$. No caso relatado, a progressão das lesões desencadeou instabilidade hemodinâmica e insuficiência respiratória, com transferência para a unidade de cuidados intensivos.

Os exames laboratoriais a serem solicitados são: hemograma com diferencial, reticulócitos, plaquetas e provas de

\section{Referências bibliográficas}

1. Cooper JG, Edwards SL, Holmes JD. Kaposiform hemangioendothelioma: case report and review of the literature. Br J Plast Surg 2002;55:163-5

2. Beardsley DS. Platelet abnormalities in infancy and childhood. In: Nathan DG, Oski FA, editors. Hematology in infancy and childhood. $4^{\text {th }}$ ed. Philadelphia: Saunders; 1993. p. 1585-6.

3. Iwami D, Shimaoka S, Mochizuki I, Sakuma T. Kaposiform hemangioendothelioma of the mediastinum in a 7-month-old boy: a case report. J Pediatr Surg 2006:41:1486-8.

4. Hervella M, Iglesias ME. Vascular tumors as syndromic indicators. An Sist Sanit Navar 2004;27 (Suppl 1):33-44.

5. Campos HGA, Curado JH. Angiodisplasias. In: Pitta GBB, Castro AA, Burihan E, editores. Angiologia e cirurgia vascular: guia ilustrado. Maceió: Uncisal/ Ecmal \& Lava; 2003. coagulação. Diante de CIVD importante, há alteração do AP e TTPA e baixos níveis de fibrinogênio ${ }^{(1,6)}$.

O tratamento consiste em cuidados de suporte, como transfusões de plaquetas, criopreciptado e plasma fresco congelado. As drogas utilizadas são prednisona e dexametasona $(2 \mathrm{mg} / \mathrm{kg} /$ dia) por, no mínimo, duas semanas, até a resolução dos sintomas. O uso de corticóide é indicado nos pacientes portadores de hemangiomas, quando há trombocitopenia, sendo, nestes casos, a primeira opção de terapia farmacológica ${ }^{(8)}$. É necessário introduzir também medicações anticoagulantes ou antiplaquetárias ${ }^{(2,3)}$. O ácido tranexâmico, empregado no presente caso, é uma droga anti-hemorrágica a ser administrada até a remissão dos hemangiomas. Diante da resposta satisfatória à terapia clínica de suporte, associada às drogas anticoagulantes e anti-hemorrágicas, o tratamento cirúrgico das lesões é dispensável.

O tratamento cirúrgico, com a ressecção completa do hemangioma, é indicado para a resolução da plaquetopenia e da CIVD não responsivas ao tratamento conservador ${ }^{(4,6)}$. Dependendo de sua localização, pode haver necessidade de cirurgia geral, torácica ou neurocirurgia para excisão ou ligação de vasos que irrigam o hemangioma. A amputação pode ser feita em lesões intratáveis envolvendo um membro. Algumas lesões são inoperáveis e a embolização é discutível para solucionar estes casos.

Os hemangiomas torácicos podem comprometer a expansibilidade pulmonar e causar insuficiência respiratória ${ }^{(2)}, \mathrm{com}^{-}$ plicação presente na criança aqui descrita. Outra complicação pode ser o sangramento secundário à CIVD não responsiva à transfusão de plaquetas, que pode levar ao óbito. Ulceração e sangramento do hemangioma também podem acontecer ${ }^{(5)}$.

O prognóstico é bom quando a causa da síndrome de Kasabach-Merritt é reconhecida e devidamente tratada. Porém, sem tratamento, a mortalidade varia de 10 a $40 \%{ }^{(4)}$.

6. Maguiness S, Guenther L. Kasabach-Merritt syndrome. J Cutan Med Surg 2002;6:335-9.

7. Deraedt K, Vander Poorten V, Van Geet C, Renard M, De Wever I, Sciot R. Multifocal kaposiform haemangioendothelioma. Virchows Arch 2006;448:843-6.

8. Serafim AP, Almeida Junior LC, Silva MT, Carvalho RB, Altemani AM. Hemangioendotelioma kaposiforme associado à Síndrome de Kasabach-Merritt. J Pediatr (Rio J) 1998;74:338-42.

9. López Gutiérrez JC, Patrón Romero M. Thoracic kaposiform hemangioendothelioma. Four consecutive cases with distinct outcome. An Pediatr (Barc) 2005;63:72-6. 